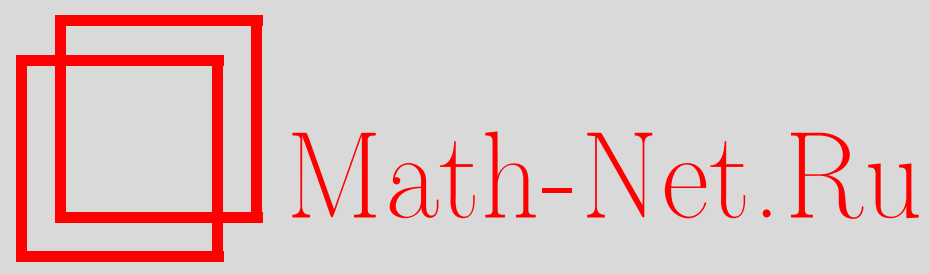

В. М. Левчук, О слабо факторизуемых группах, Матем. заметки, 2003, том 73, выпуск 4, 565-572

DOI: https://doi.org/10.4213/mzm204

Использование Общероссийского математического портала Math-Net.Ru подразумевает, что вы прочитали и согласны с пользовательским соглашением http://www .mathnet.ru/rus/agreement

Параметры загрузки:

IP: 34.227 .88 .159

26 апреля 2023 г., 10:50:57

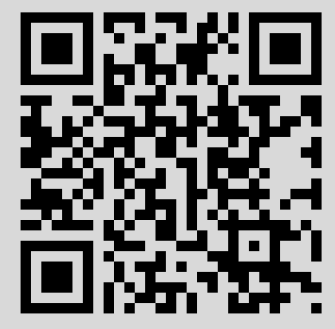




\section{О СЛАБО ФАКТОРИЗУЕМЫХ ГРУППАХ}

\section{В.М. Левчук}

Группы с дополняемыми подгруппами, назьваемые вполне факторизуемыми, изучены в работах $\Phi$. Холла, С. Н. Черникова и Н. В. Черниковой (Баевой). Для вполне факторизуемости достаточно (теорема 1), чтобы всякая собственная подгруппа имела нормальное дополнение в некоторой большей подгруппе. Группа называется слабо факторизуемой, если каждая ее собственная подгруппа дополняема в некоторой большей подгруппе; в "Коуровской тетради" поставлен вопрос 8.31 об описании таких конечных групп. Рассматриваются некоторые их свойства. Вопрос исследуется для силовских $p$-подгрупп групп Шевалле типа харак теристики $p$. Основная теорема 2 устанавливает слабую факторизуемость силовских $p$-подгрупп в симметрической и знакопеременной группах подстановок и в классических линейных группах над полями характеристики $p>0$, исключая унитарные группы нечетной размерности $>p$.

Библиографоия: 16 названий.

Введение. Подгрупу $M$ назьваем дополняемой в групе $G$, если в $G$ существует дополнение к ней, т.е. подгруппа $K$ такая, что $M \cap K=1$ и $G=M K(=K M)$. Группу называют вполне факторизуемой, если в ней дополняема любая подгрупша. Такие группы изучены в работах $\Phi$. Холла [1], С.Н. Черникова [2] и Н. В. Черниковой (Баевой) [3], [4]. Близкие к ним группы исследовали и применяли Ю.М. Горчаков и др., см. [5], [6].

Для вполне факторизуемости группы достаточно, чтобы всякая ее собственная подгруппа обладала нормальным дополнением в некоторой большей подгруппе (теорема 1). Освобождаясь от условия нормальности дополнения, приходим к существенно более широкому классу групп: групу назьваем слабо факторизуемой, если любая ее собственная подгруппа дополняема в некоторой большей подгруппе. Вопрос об описании таких конечных групп записан в [7, вопрос 8.31]. К ним относятся, например, простая группа $P S L_{2}(7)$, диэдральные 2-группы и конечные группы простого показателя $p$; существенность здесь условия конечности показьвает пример А. Ю. Ольшанского [8, теорема 28.1] бесконечной группы показателя $p$ с максимальными подгруппами простых порядков. Некоторые свойства слабо факторизуемых групп рассматриваются в п. 1 . Слабую факторизуемость полупрямого произведения вполне факторизуемой и слабо факторизуемой групп устанавливает лемма 1.

Основная в работе теорема 2 выявляет слабую факторизуемость силовских $p$-подгрупп в симметрической и знакопеременной группах подстановок любой конечной степени и в классических линейньх группах надполем характеристики $p>0$, исключая унитарные группы нечетной размерности $>p$. С другой стороны, силовские 2-подгруппы 
в скрученных групшах Шевалле типов ${ }^{2} B_{2},{ }^{2} F_{4}$ и ${ }^{2} A_{2 n}$ над полем характеристики 2 и силовские 3 -подгруппы групп Ри типа ${ }^{2} G_{2}$ надполем характеристики 3 обладают собственной подгруппй, пересекающейся нетривиально с каждой неединичной подгрупшой (предложение 1). См. также замечание в конце п. 2.

1. Вполне факторизуемые и слабо факторизуемые группы. Свойства полной и слабой факторизуемости групшы очевидно наследуются на фактор-группы. Полная факторизуемость наследуется и на подгрупы, как показьвает

Лемма (С.Н. Черников [2]). Пусть $G$ - группа, $H, M$ u $R$ - такие еe подгруппы, что $H \supseteq M u R$ - дополнение подгруппь $M$ в $G$. Тогда $R \cap H$ - дополнение nодгруппь $M$ в $H$.

Отметим, что если $D$ есть дополнение к подгруппе $M$ в какой-либо подгруппе и элемент $g$ лежит в произведении $N_{G}(D) N_{G}(M)$ нормализаторов подгруп $M$ и $D$ в группе $G$, то $M\left(g^{-1} D g\right)$ есть подгрупша и $g^{-1} D g$ является в ней дополнением к $M$.

ТЕорема 1. Для произвольной группы $G$ әквивалентны следующие условия:

(i) всякая собственная подгруппа группь $G$ обладает нормальным дополнением в некоторой большей подгруппе;

(ii) всякая подгруппа группь $G$ обладает нормальным дополнением в $G$;

(iii) әруппа $G$ является абелевой вполне факторизуемой группой.

ДокАЗАТЕЛьСтво. Достаточно доказать утверждения (i) $\rightarrow$ (ii) и (ii) $\rightarrow($ iii), поскольку утверждение (iii) $\rightarrow$ (i) очевидно.

(i) $\rightarrow$ (ii). Пусть $H$ - собственная подгруппа групшы $G$. Положим $H_{0}=H$. Допустим, что для некоторого порядкового числа $\alpha>0$ подгруппы $H_{\beta}$ для всех $\beta<\alpha$ уже определены и порождают в $G$ собственную подгруппу. Тогда нормальное дополнение к последней, выбранное произвольно в некоторой большей подгруппе группы $G$, обозначаем через $H_{\alpha}$. Таким образом, получаем последовательность подгрупा $H_{0}, H_{1}, \ldots, H_{\alpha}$, $\ldots$, которые порождают группу $G$.

По построению $H_{\alpha} \triangleleft H_{\beta} H_{\alpha}$ при всех $\beta<\alpha$. Поэтому подгрупша $N$, порожденная подгруппами $H_{\alpha}$ для всевозможных $\alpha>0$, нормальна в $G$. Ясно, что $G=H N$. Если $t-$ произвольный элемент пересечения $H \cap N$, то он записьвается в виде конечного произведения $t=h_{1} h_{2} \cdot \ldots \cdot h_{k}$, где $k \geqslant 1$ - натуральное число, $h_{i} \in H_{\alpha_{i}}$ и $0<\alpha_{1}<\alpha_{2}<\cdots<\alpha_{k}$. Поэтому

$$
h_{k}=\left(h_{1} h_{2} \cdot \ldots \cdot h_{k-1}\right)^{-1} t \in H_{\alpha_{k}} \cap\left(H_{0} H_{\alpha_{1}} H_{\alpha_{2}} \cdot \ldots \cdot H_{\alpha_{k-1}}\right)=1 .
$$

Следовательно, $h_{k}=1$ и аналогично $h_{1}=h_{2}=\cdots=h_{k-1}=1$, так что $t=1$. Таким образом, $H \cap N=1$ и $N$ - нормальное дополнение подгруппы $H$ в группе $G$.

$(i i) \rightarrow($ iii $)$. Докажем абелевость произвольной группы $G$ с условием $(i i)$. Поскольку $G$ - вполне факторизуемая и, следовательно, периодическая группа [4], то при $G \neq 1$ она должна содержать подгруппу простого порядка. В силу $(i i)$ группа $G$ имеет также и нормальную подгруппу простого индекса; нормальное дополнение к ней, очевидно, лежит в центре группы $G$. Таким образом, в любой неединичной группе с условием $(i i)$ центр неединичен.

Предположим, что группа $G$ не совпадает со своим центром $Z(G)$. Выберем в ней какое-либо нормальное дополнение $H$ к центру $Z(G)$. Пусть $K$ - произвольная подгруппа 
в $H$, и $R$ - какое-либо ее нормальное дополнение в $G$. Тогда $R \cap H$ есть дополнение к подгруппе $K$ в $H$ по лемме Черникова. С другой стороны, $(R \cap H) \triangleleft H$. Следовательно, группа $H$ также удовлетворяет условию $(i i)$ и по доказанному ее центр $Z(H)$ неединичен. Однако, $G=Z(G) \times H$ и поэтому $Z(H)=Z(G) \cap H=1$. Полученноепротиворечие доказьвает равенство $H=1$ и абелевость $G$. Теорема доказана.

Известно, что абелевы вполне факторизуемые групш - это в точности прямые произведения циклических групп простых порядков. Полностью вполне факторизуемые группы изучены в работах [1]-[4]. Их описьвает

Теорема [4]. Группа $G$ тогда и только тогда вполне факторизуема, когда она представима в виде полупрямого произведения $G=H 入 N$ подгрупп $H$ u $N$, раскладывающихся в прямое произведение ииклических групп простых порядков, причем все мнохители хотя бы одного разложения группы $H$ инвариантны в группе $G$.

Следующая лемма указывает метод построения слабо факторизуемьх групп из вполне факторизуемых групп.

ЛЕмма 1. Полупрямое произведение $F \lambda K$ вполне факторизуемой группь $F u$ слабо факторизуемой группы $K$ есть слабо факторизуемая группа.

ДокАЗАТЕЛЬСТво. Пусть $L-$ собственная подгруппа группы $F \lambda K$. Если $L \supseteq F$ и $D$ - дополнение к $L \cap K$ в какой-либо подгруппе в $K$, то $L D=(F \lambda(L \cap K)) D-$ подгруппа и $D$ дополняет в ней подгрупу $L$. Допустим, что $L \nsupseteq F$. Тогда пересечение $L \cap F$ обладает неединичным дополнением $D$ в $F$, причем

$$
D \cap L=(D \cap F) \cap L=D \cap(F \cap L)=1, \quad D L=D(F \cap L) L=F L .
$$

Поэтому подгруппа $L$ дополняема в $F L$ подгруппой $D$. Лемма доказана.

По аналогии с доказательством теоремы 1 показывается, что разрешимая подгруппа произвольной групшы $G$ всегда лежит в разрешимой подгрупше $H$, которая не имеет разрешимого нормального дополнения в больших подгруппах. Произвольное нормальное дополнение к $H$ в большей подгруппе в этом случае имеет единичное пересечение с каждой разрешимой подгруппой, содержащей $H$.

Условие слабой факторизуемости нильпотентной группы, очевидно, равносильно тому, что любая ее собственная подгруппа дополняема и нормальна в некоторой большей подгруппе. Так, всякая конечная группа простого показателя $p$ слабо факторизуема; существенность здесь условия конечности показьвает пример А. Ю. Ольшанского [8, теорема 28.1] бесконечной групшы с максимальными подгруппами простого порядка $p$.

Лемма 2. Диәдральная группа слабо факторизуема тогда и только тогда, когда ее порядок конечен и не делится на квадраты простых нечетных чисел.

ДокАЗАТЕЛЬСТВо. В диэдральной 2-группе нормализатор любой собственной подгруппы $L$ является также диэдральной подгруппой порядка $\geqq 2|L|$ и, следовательно, с числом $>|L|$ инволюций; отсюда $L$ имеет дополнение в подгрупше порядка $2|L|$. Поэтому в произвольной диэдральной группе $G$ (или в группе, порождаемой двумя инволюциями) силовская 2-подгруппа $G_{2}$ является слабо факторизуемой группой. Группа $G$ имеет циклическую подгруппу $A$ индекса 2 . Кроме того, $G=B \lambda G_{2}$, где $B$ есть 
2 -дополнение в $A$ при $|A|<\infty$, и $B=A$ в противном случае. Если $B$ - вполне факторизуемая группа, то $G$ есть слабо факторизуемая группа по лемме 1 . Очевидно, вполне факторизуемость циклической группы равносильна требованию, что ее порядок конечен и не делится на квадраты простых чисел.

Допустим сейчас, что для некоторого простого числа $p>2$ индекс подгруппы $B^{p}=$ $\left\{b^{p} \mid b \in B\right\}$ в $B$ равен $p$. Тогда $M=B^{p} \lambda G_{2}$ есть максимальная подгруппа индекса $p$ в $G$. Предположим, что в $G$ существует дополнение $D$ к $M$. Поскольку $D \subseteq A$ и $D \cap B^{p}=1$, должны иметь $|A|<\infty$ и $|D|=p$. Силовские $p$-подгрупшы групп $B$ и $G$ содержатся в $A$. Поэтому они нормальны в $G$ и совпадают. Более того, они совпадают с $D$ в силу цикличности групшы $B$ и условия $D \cap B^{p}=1$. Но тогда получаем противоречие с условием $\left|B: B^{p}\right|=p$. Таким образом, в группе $G$ максимальная подгруппа $M$ не имеет дополнения. Лемма доказана.

В диэдральной 2-группе циклическая подгруппа порядка $>2$ не является слабо факторизуемой группой. Таким образом, свойство слабой факторизуемости группы не наследуется подгрупшами. С другой стороны, очевидна

Лемма 3. Конечная группа является слабо факторизуемой, если все ее максимальные подгруппы дополняемы и слабо факторизуемы.

ПримеР 1. Знакопеременная группа подстановок 4-й степени слабо факторизуема, поскольку ее максимальные подгруппы исчерпываются силовскими подгруппами, которые дополняемы и элементарные абелевы. Помимо нее в симметрической группе $\mathscr{S}_{4}$ подстановок 4-й степени максимальными являются диэдральные подгруппы порядков 6 и 8, которые дополняемы циклическими подгруппами. Следовательно, $\mathscr{S}_{4}$ является слабо факторизуемой группой. (Группа $\mathscr{S}_{5}$ этим свойством не обладает, поскольку ее диэдральная подгруппа порядка 12 максимальна и имеет общую инволюцию с любой подгруппой порядка 10; см. также [9]). Группа $P S L_{2}(7)$ порядка 168 имеет два класса сопряженных подгрупп, изоморфных $S_{4}$ (параболические подгрупшы в группе $\left.P S L_{3}(2) \simeq P S L_{2}(7)\right)$; все они дополняемы подгруппой порядка 7. Остальные ее максимальные подгруппы дают нормализаторы силовских 7-подгруп, имеющие порядок 21 и дополняемые силовской 2-подгруппой. Таким образом, простая группа $P S L_{2}(7)$ также слабо факторизуема. Как несложно вытекает из [10], среди простых групп $P S L_{2}(q)$ нет других слабо факторизуемых групш.

2. Силовские подгруппы классических групп. Классические линейные группы ниже рассматриваются как грушы Шевалле классических лиевых типов $A_{n}, B_{n}$, $C_{n}, D_{n},{ }^{2} A_{n}$ и ${ }^{2} D_{n}$. Следуюшая, основная в работе, теорема выявляет склонность к слабой факторизуемости силовских $p$-подгрупп классических групा.

ТЕОРема 2. Силовские р-подгруппы в симметрической и знакопеременной группах подстановок любой конечной степени и в группах Шевалле классических типов $\neq{ }^{2} A_{2 n}$ над полем характеристики $p>0$ являются слабо факторизуемыми группами.

\section{Нам потребуется}

Лемма 4. Силовская р-подгруппа симметрической группь подстановок степени $p^{n}, n \geqslant 1$, представима в виде

$$
V_{n} \lambda\left(V_{n-1} \lambda\left(\ldots\left(V_{3} \lambda\left(V_{2} \lambda V_{1}\right)\right) \ldots\right)\right)
$$


для подходящих әлементарных абелевых р-подгрупп $V_{1}, V_{2}, \ldots, V_{n}$.

ДокАЗАТЕЛЬСтво. Пусть $p$ - простое число и $P_{n}$ - силовская $p$-подгруппа симметрической группы подстановок степени $p^{n}, n \geqslant 1$. Групша $P_{n}$ построена в $[11 ; 5.9]$ и $[12$; 11.3.1] как сплетение $n$ циклических групп преобразований,

$$
P_{n}=\left(\ldots\left(\left(Z_{p} \succ Z_{p}\right) \curlywedge Z_{p}\right) \ldots\right) \succ Z_{p}
$$

в частности, $\left|P_{n}\right|=p^{1+p+p^{2}+\cdots+p^{n-1}}$. Ее представление вида (1) дает ассоциативность операции сплетения групп преобразований $[12 ; 6.2 .13]$, показьвающая, что

$$
P_{n}=Z_{p} \curlywedge\left(Z_{p} \curlywedge\left(Z_{p} \curlywedge\left(\ldots\left(Z_{p} \prec Z_{p}\right) \ldots\right)\right)\right) .
$$

(В классе групп операции полного и дискретного сплетений неассоциативны [12; 6.2.7].) Сомножители $V_{j}$ можно записать даже явно. Рассмотрим $\left(p^{t} \times p\right)$-матрищу

$$
A_{t 1}=\left[\begin{array}{ccccc}
1 & 1+p^{t} & 1+2 p^{t} & \ldots & 1+(p-1) p^{t} \\
2 & 2+p^{t} & 2+2 p^{t} & \ldots & 2+(p-1) p^{t} \\
3 & 3+p^{t} & 3+2 p^{t} & \ldots & 3+(p-1) p^{t} \\
\vdots & \vdots & \vdots & \ddots & \vdots \\
p^{t} & 2 p^{t} & 3 p^{t} & \ldots & p^{t+1}
\end{array}\right]
$$

Прибавляя к каждому ее элементу число $(k-1) p^{t+1}$, получаем матрицу, обозначаемую через $A_{t k}\left(0 \leqslant t<n, 1 \leqslant k \leqslant p^{n-t-1}\right)$. Пусть $\beta_{t k}$ есть подстановка, в циклической записи которой циклами являются строки матрицы $A_{t k}$. Для фиксированного $t$ всевозможные подстановки $\beta_{t k}$ перестановочны (так как действуют на непересекающихся множествах) и, следовательно, порождают элементарную абелеву $p$-подгрупш порядка $p^{p^{n-t-1}} ;$ ее обозначим через $V_{n-t}$. С другой стороны,

$$
\beta_{t k}^{-1} \beta_{i m} \beta_{t k}=\beta_{i m^{\prime}}, \quad 0 \leqslant i<t<n,
$$

где $m^{\prime}$ однозначно определяется условиями

$$
(i-1) p^{t-i}<m^{\prime} \leqslant i p^{t-i}, \quad m^{\prime} \equiv m+p^{t-i-1} \bmod p^{t-i}
$$

Отсюда вытекает, что подгруппы $V_{j}, 1 \leqslant j \leqslant n$, порождают подгруппу (1), которая совпадает с $P_{n}$ в силу равенства порядков. Лемма доказана.

ЛЕмма 5. Прямое произведение конечного числа групп вида (1), построенных из әлементарных абелевых р-подгрупп, есть такхсе группа вида (1), построенная из подходяших әлементарных абелевых р-подгрупп. 
ДоКАЗАТЕЛЬСТВо. Простая индукция сводит общий случай к случаю двух групп вида (1).

Пусть $U$ и $V$-группы вида (1), построенные по элементарным абелевым $p$-подгруппам $U_{1}, U_{2}, \ldots, U_{m}$ и, соответственно, $V_{1}, V_{2}, \ldots, V_{n}$. Допустим, что $1 \leqslant m \leqslant n$. Положим $U_{k}=1$ при $k<1$. Тогда прямое произведение $W=U \times V$ есть группа вида (1), построенная по элементарным абелевым $p$-подгруппам $W_{j}=U_{j+m-n} \times V_{j}, 1 \leqslant j \leqslant n$. Лемма доказана.

Докажем сейчас теорему 2 для силовских подгрупп в симметрической и знакопеременной группах подстановок.

Пусть $p$ - простое число и $P$ - силовская $p$-подгруппа симметрической группы $\mathscr{S}_{k}$ подстановок произвольной конечной степени $k$. Известно (см. $[11 ; 5.9]$ и $[12 ; 11.3 .1])$, что группа $P$ изоморфна прямому произведению силовских $p$-подгрупп симметрических групп подстановок $p$-примарных степеней. Поэтому в силу лемм 4 и $5 P$ есть группа вида (1) для подходящих элементарньх абелевых $p$-подгрупп $V_{1}, V_{2}, \ldots, V_{n}$. Индукция по $n$ и лемма 1 показывают, что такая группа всегда является слабо факторизуемой.

Ясно, что $P$ есть силовская $p$-подгруппа и в знакопеременной группе $\mathscr{A}_{k}$ при $p>2$. Допустим, что $p=2$. Тогда пересечение $\mathscr{A}_{k} \cap P$ есть силовская 2 -подгруппа в $\mathscr{A}_{k}$. В силу леммы 5 и по построению подгрупп $V_{i}$ в лемме 4 можно считать, что каждая из них при $i<n$ порождается подстановками с четньм числом циклов одной длины. Поэтому все подгрупшы $V_{1}, V_{2}, \ldots, V_{n-1}$ лежат в $\mathscr{A}_{k} \cap P$. По лемме Черникова подгруппа $\left(\mathscr{A}_{k} \cap P\right) \cap V_{n}$ есть дополнение к подгруппе $V_{n-1} \lambda\left(\ldots\left(V_{3} \lambda\left(V_{2} \lambda V_{1}\right)\right) \ldots\right)$ в $\mathscr{A}_{k} \cap P$. Поскольку $\mathscr{A}_{k} \cap V_{n}=\left(\mathscr{A}_{k} \cap P\right) \cap V_{n} \unlhd\left(\mathscr{A}_{k} \cap P\right)$, должны иметь

$$
\mathscr{A}_{k} \cap P=\left(\mathscr{A}_{k} \cap V_{n}\right) \lambda\left(V_{n-1} \lambda\left(\ldots\left(V_{3} \lambda\left(V_{2} \lambda V_{1}\right)\right) \ldots\right)\right)
$$

Следовательно, силовские подгрупшы знакопеременной группы подстановок также слабо факторизуемы.

Рассмотрим далее случай групп Шевалле. Через $\Phi$ обозначим приведенную систему корней простой комплексной алгебры Ли, через $\Phi^{+}-$систему ееположительных корней. Группа Шевалле $G(K)$ нормального типа $G=\Phi$ или скрученного типа $G={ }^{2} \Phi$ над полем $K$ порождается корневыми подгруппами $X_{a}(a \in G)$; см. [13]. Ее максимальная унипотентная подгруппа $U G(K)=\left\langle X_{a} \mid a \in G^{+}\right\rangle$при $\operatorname{char} K=p>0$ есть силовская $p$-подгрупша.

Лемма 6. Унипотентная подгруппа $U G(K)$ любого из типов

$$
G=A_{n}, B_{n}, C_{n}, D_{n+1} \quad(n>2), \quad{ }^{2} D_{n+1},{ }^{2} A_{2 n-1}, E_{n+1} \quad(n=5,6)
$$

над полем $K$ характеристики $p>0$ представима в виде (1) для подходящих әлементарных абелевых $p$-подгрупп $V_{1}, V_{2}, \ldots, V_{n}$.

ДокАЗАТЕЛЬСтво. По определению подгрупша $T(r)$ групшы $U \Phi(K), r \in \Phi^{+}$, порождается корневыми подгруппами $X_{a}\left(a \in \Phi^{+}\right)$, для которых $r-a$ есть линейная комбинация простых корней с неотрицательньми коэффициентами. Ясно, что определение этой подгруппы имеет смысл и для скрученных групп, причем $T(r) \unlhd U G(K)$.

Пусть сейчас подгруппа $T(r)$ групшы $U \Phi(K)$ выбрана для простого корня $r$, который в обозначениях $[14$, таблицы I-IX] является первым в графе Кокстера системы корней $\Phi$ 
для лиевых типов $B_{n}$ и $D_{n}$, а в остальных случаях - последним. В частности, $r$ для лиевых типов $B_{n}$ и $C_{n}$ - это длинньй простой корень, крайний в графе Кокстера. В скрученных группах типов ${ }^{2} A_{2 n-1}$ и ${ }^{2} D_{n+1}$ подгруппу $T(r)$ выбираем, как обычно, по аналогии с типами $C_{n}$ и $B_{n}$, соответственно; см. также [13, 13.3 .1 и 13.3.2]. Таким образом, в представлении $[15, \S 2]$ групшы $U G(K)$ (ее представление в $[15] \Phi^{+}$-матрицами для классических типов $G$ основано на том, что каждому положительному корню вида $e_{i}-t e_{j}(i \geqslant j, t \in\{0,-1,+1\})$ сопоставляется элементарная $\Phi^{+}$-матрица $\left.e_{i, t j}\right)$ подгрупше $T(r)$ для лиевых типов $B_{n}, D_{n}$ и ${ }^{2} D_{n+1}$ соответствует подгрупша $T_{n n-1}$, а для лиевых типов $C_{n}$ и ${ }^{2} A_{2 n-1}-$ подгруппа $T_{1,-1}$. С использованием лемм $3-5$ [15] и таблиц V-VII [14] несложно убедиться, что в групе $U G(K)$ корневые подгрупш, не входящие в $T(r)$, порождают дополнение к $T(r)$ и, кроме того,

$$
\begin{gathered}
U C_{n}(K)=T(r) \lambda U A_{n-1}(K), \quad U^{2} A_{2 n-1}(K)=T(r) \lambda U A_{n-1}(K), \\
U E_{n}(K)=T(r) \lambda U E_{n-1}(K) \quad(n=7,8), \quad U E_{6}(K)=T(r) \lambda U D_{5}(K) ;
\end{gathered}
$$

для лиевых типов $A_{n}, B_{n}, D_{n}$ и $^{2} D_{n+1}$ дополнение является унипотентной подгруппой группы Шевалле типа $A_{n-1}, B_{n-1}, D_{n-1}$ и $^{2} D_{n}$, соответственно, т.е. одноименного типа, но меньшего лиева ранга.

Во всех перечисленных случаях, исключая тип $E_{8}$, выбранная подгрушпа $T(r)$ является прямым произведением порождающих ее корневых подгрупп. То же самое верно и для подгрупп $U A_{1}(K)\left(\simeq U B_{1}(K) \simeq U^{2} D_{2}(K)\right)$ и $U D_{2}(K)$, т.е. типа $A_{1} \cdot A_{1}$. При $G \neq{ }^{2} A_{2 n},{ }^{2} B_{2},{ }^{2} G_{2},{ }^{2} F_{4}$ корневые подгруппы групшы $U G(K)$ изоморфны аддитивной группе основного поля $K$ или (в случае скрученных групп) его определенного подполя; см. $[13,13.6 .4]$. Поэтому в условиях леммы подгрупа $T(r)$ является элементарной абелевой $p$-подгруппой, и мы полагаем $V_{n}=T(r)$. Требуемое в лемме представление $(1)$ группы $U G(K)$ сейчас получаем индукцией по лиеву рангу $n$. Лемма доказана.

Леммы 6 и 1 доказывают теорему для случая групп Шевалле. Таким образом, теорема 2 полностью доказана.

Силовские $p$-подгруппы в группах Шевалле типов $E_{6}$ и $E_{7}$ над полем характеристики $p>0$ слабо факторизуемы в силу лемм 6 и 1 . Исключительная для теоремы 2 и леммы 6 группа $U^{2} A_{2 n}(K)$ при $\operatorname{char} K=p>2 n$ есть нильпотентная группа показателя $p$ и поэтому также слабо факторизуема. В то же время, ограничение $G \neq^{2} A_{2 n}$ в теореме 2 существенно, как показьвает

ПРЕДЛОЖЕНИЕ 1. Силовские 2-подгруппь в скрученных группах Шевалле типов ${ }^{2} B_{2},{ }^{2} F_{4} u^{2} A_{2 n}$ над полем характеристики 2 и силовские 3-подгруппь групп Ри типа ${ }^{2} G_{2}$ обладают собственной подгруппой, пересекающейся нетривиально $c$ любой неединичной подгруппой.

ДокАЗАтЕльСтво. В группах $U^{2} A_{2}(K)$ и $U^{2} B_{2}(K)$ над полем $K$ характеристики 2 квадрат любого нецентрального элемента является неединичным элементом центра, как следует из $[13,13.6 .4$ (iv), (vi)]. Поэтому их центр (равносильно, коммутант или подгруппа Фраттини) пересекается нетривиально с любой неединичной подгруппой. Используя $[13,13.6 .4$ (vii)] (см. также [16]), то же самое получаем для коммутанта группы $U^{2} G_{2}(K)$ над полем $K$ характеристики 3.

Пусть $U=U^{2} \Phi(K)$, где $K$ - поле характеристики 2 , а $\Phi$ - типа $A_{2 n}$ или $F_{4}$. Тогда в $\Phi$ существует и определен однозначно фундаментальньй класс $S$ эквивалентности 
типа $A_{2}$ или $B_{2}$. Как и в $[13,13.6 .4(\mathrm{iv}),(\mathrm{vi})]$, ему соответствует в $U$ корневая подгруппа $X_{S}$, изоморфная $U^{2} A_{2}(K)$ или соответственно $U^{2} B_{2}(K)$; см. также [15]. Остальные корневые подгруппы в групе $U$ порождают нормальную подгруппу $U_{S}$. Подгрупу, которую порождают $U_{S}$ и центр подгруппы $X_{S}$, обозначим через $M$. Пусть $\alpha \in U \backslash M$. Тогда $\alpha$ по модулю $U_{S}$ есть нецентральньй элемент подгрупшы $X_{S}$ и по доказанному его квадрат не может лежать в $U_{S}$. Отсюда вытекает, что подгруппа $M$ пересекается нетривиально с любой неединичной подгруппой группы $U$. Это завершает доказательство.

Поскольку всякая конечная группа изоморфно вкладьвается в симметрическую группу подстановок подходящей конечной степени, то теорема 2 дает

СлЕДСТВИЕ. Всякая конечная р-группа изоморфно вкладывается в слабо факторизуемую конечную р-группу.

В связи с вопросом 8.31 [7] сделаем два замечания. Прежде всего, заметим, что предыдущее следствие еще не исключает возможность эффективного описания конечнњх слабо факторизуемых $p$-групп. Например, не выявлено, какую часть среди них занимают все $p$-группы, получаемые кратным применением леммы 1 из вполне факторизуемых $p$-групп и групп показателя $p$. Отметим также предположение о том, что группа $P S L_{2}(7)$ является единственной слабо факторизуемой группой из конечных простых неабелевых групп.

\section{СПИСОК ЦИТИРОВАННОЙ ЛИТЕРАТУРЫ}

[1] Hall Ph. Complemented groups // J. London Math. Soc. 1937. V. 12. P. 201-204.

[2] Черников С. Н. Группы с системами дополняемых подгрупп // Матем. сб. 1954. Т. 35 (77). № 1. C. 93-128.

[3] Баева Н.В. Вполне факторизуемые группы // Докл. АН СССР. 1953. Т. 92. №5. C. $877-880$.

[4] Черникова Н. В. Группы с дополняемыми подгруппами // Матем. сб. 1956. Т. 39 (81). № 3. C. $1202-1220$.

[5] Горчаков Ю. М. Группы с конечными классами сопряженных элементов. М.: Наука, 1978.

[6] Черников С.Н. Группы с заданными свойствами системы подгрупп. М.: Наука, 1980.

[7] Коуровская тетрадь (нерешенные вопросы теории групп). Изд. 14-е. Новосибирск: ИМ СО PAH, 1999.

[8] Ольшанский А. Ю. Геометрия определяющих соотношений в группах. М.: Наука, 1989.

[9] Thompson J. G. Hall subgroups of the symmetric groups // J. Combin. Theory. 1966. V. 1. № 2. P. 271-279.

[10] Ito N. On the factorizations of the linear fractional group $L F\left(2, p^{n}\right) / /$ Acta Sci. Math. 1953. V. 15. №1. P. 79-84.

[11] Холл М. Теория групп. М.: ИЛ, 1962.

[12] Каргаполов М.И., Мерзляков Ю. И. Основы теории групп. 4-е изд.. М.: Наука, 1996.

[13] Carter R. W. Simple Groups of Lie Type. London: J. Wiley, 1972.

[14] Бурбаки Н. Группы и алгебры Ли. Гл. IV-VI. М.: Мир, 1972.

[15] Левчук В. М. Автоморфизмы унипотентных подгрупп групп Шевалле // Алгебра и логика. 1990. Т. 29. № 3. С. 315-338.

[16] Левчук В. М., Нужин Я.Н. О строении групп Ри // Алгебра и логика. 1985. Т. 24. № 1. C. 26-41. 